Article

\title{
Investigation of the InAs/GaAs Quantum Dots' Size: Dependence on the Strain Reducing Layer's Position
}

\section{Manel Souaf ${ }^{1}$, Mourad Baira ${ }^{1}$, Olfa Nasr ${ }^{1}$, Mohamed Helmi Hadj Alouane ${ }^{1}$, Hassen Maaref ${ }^{1}$, Larbi Sfaxi ${ }^{1}$ and Bouraoui Ilahi ${ }^{2,1, *}$}

1 Laboratoire de Micro-Optoélectronique et Nanostructures, Faculté des Sciences, Avenue de l'environnement, Université de Monastir, Monastir 5019, Tunisia; E-Mails: manelsouaf@yahoo.fr (M.S.); mourad.baira@isimm.rnu.tn (M.B.); olfaa.nasr@gmail.com (O.N.); helmi.alouane@yahoo.fr (M.H.H.A); hassen.maaref@fsm.rnu.tn (H.M.); larbi.sfaxi@fsm.rnu.tn (L.S.)

2 King Saud University, Department of Physics \& Astronomy, College of Sciences, P.O. 2455, Riyadh 11451, Saudi Arabia

* Author to whom correspondence should be addressed; E-Mail: bilahi@ksu.edu.sa; Tel.: +966-11-467-6393; Fax: +966-11-467-3656.

Academic Editor: Sanjaya Senanayake

Received: 4 June 2015 / Accepted: 13 July 2015 / Published: 24 July 2015

\begin{abstract}
This work reports on theoretical and experimental investigation of the impact of InAs quantum dots (QDs) position with respect to InGaAs strain reducing layer (SRL). The investigated samples are grown by molecular beam epitaxy and characterized by photoluminescence spectroscopy (PL). The QDs optical transition energies have been calculated by solving the three dimensional Schrödinger equation using the finite element methods and taking into account the strain induced by the lattice mismatch. We have considered a lens shaped InAs QDs in a pure GaAs matrix and either with InGaAs strain reducing cap layer or underlying layer. The correlation between numerical calculation and PL measurements allowed us to track the mean buried QDs size evolution with respect to the surrounding matrix composition. The simulations reveal that the buried QDs' realistic size is less than that experimentally driven from atomic force microscopy observation. Furthermore, the average size is found to be slightly increased for InGaAs capped QDs and dramatically decreased for QDs with InGaAs under layer.
\end{abstract}


Keywords: modeling; quantum dots; strain reducing layer

\section{Introduction}

InAs self-assembled QDs formed by Stranski-Krastanov growth mode have been a subject of intense research activity towards the development of innovative devices such as QDs lasers [1,2], light emitters and detectors [3-6]. More recently, this kind of QDs has been found to be promising for photovoltaic applications [7]. However, the efficient exploitation of the self-assembled QDs towards high performance devices relay on the best optimization of their size dispersion and control of their emission energy. Accordingly, it is important to understand the impact of the capping and/or the underling layer matrix composition on the evolution of the QDs size and electronic structure. Several methods, based on the strain engineering, have been reported [8-10]. Indeed, replacing the GaAs surrounding material by InGaAs alloy leads to partial strain relief-induced modification of confinement potential allowing to manipulate inter and intraband transition energies in InAs QDs $[11,12]$. The SRL has been employed as a QDs capping layer [13], underling layer [14], or a combination between these two approaches [15]. Despite the numerous theoretical and experimental reports on the impact of the SRL on the optical and structural properties of the QDs [16-23], less attention has been devoted to the combined theoretical and optical spectroscopy investigation of the impact of the InAs QDs position with respect to the SRL on the evolution of the buried dots' size. Actually, it is well established that the buried InAs QDs undergo a compositional change duo to In segregation and in-situ intermixing [20,24,25]. Accordingly, the accurate modeling of the QDs electronic properties requires the knowledge of the exact In compositional profile [23,26]. In the meanwhile, with the absence of such data, the combination of photoluminescence spectroscopy and numerical calculation of the InAs QDs' electronic structure could constitute an alternative solution allowing a comprehensive investigation of the asymptotic buried dots' size evolution depending on the surrounding materials composition.

In this context, we report on a numerical and experimental investigation of the effect of strain reducing layer position on the size and electronic properties of the InAs/GaAs QDs system. The theoretical estimation explains well the observed experimental results.

\section{Results and Discussion}

Solving the Schrödinger equation with single band effective mass approximation by using the finite element methods (FEM) provided by COMSOL Multiphysics software, [27] affords a numerical estimation of the energy levels and corresponding wave functions. In this calculation, the InAs QDs are considered to be lens shaped as shown by the Figure 1 .

The Schrödinger equation in cylindrical coordinates is given by:

$$
\frac{-\hbar^{2}}{2 m^{*}}\left(\frac{\partial^{2}}{\partial r^{2}}+\frac{1}{r} \frac{\partial}{\partial r}+\frac{1}{r^{2}} \frac{\partial^{2}}{\partial \theta^{2}}+\frac{\partial^{2}}{\partial z^{2}}\right) \psi(r, \theta, z)+V \psi(r, \theta, z)=E \psi(r, \theta, z)
$$

where $E$ represents the electron (hole) energy of state, $m^{*}$ electron (hole) effective masse, $\hbar$ the reduced Planck constant, $\theta$ is the azimuthal angle varied from 0 to $2 \pi, r$ and $z$ are, respectively, the radial and 
axial coordinates. Due to the rotational symmetric of the system, the Schrödinger equation can be reduced to a two dimensional problem were $\psi(r, \theta, z)$ can be written in the following form:

$$
\psi(\mathrm{r}, \phi, \mathrm{z})=\chi(\mathrm{r}, \mathrm{z}) \Phi(\theta)
$$

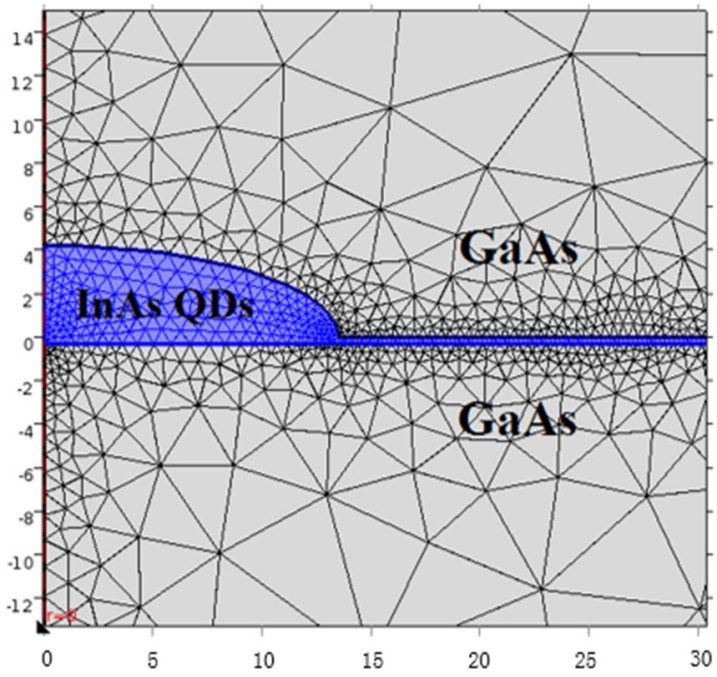

Figure 1. Schematic presentation of the zoomed lens shaped InAs quantum dots (QD) in a GaAs matrix. Also shown is the adaptive mesh refinement around the QD and the wetting layer.

By dividing Equations (1) by (2) and then multiplying by $r^{2}$, we obtain:

$$
\begin{gathered}
\frac{-\hbar^{2}}{2 m^{*}}\left(\frac{r^{2}}{\chi(\mathrm{r}, \mathrm{z})} \frac{\partial^{2} \chi(\mathrm{r}, \mathrm{z})}{\partial r^{2}}+\frac{r}{\chi(\mathrm{r}, \mathrm{z})} \frac{\partial \chi(\mathrm{r}, \mathrm{z})}{\partial r}++\frac{\partial^{2} \chi(\mathrm{r}, \mathrm{z})}{\partial z^{2}}\right)+(V-E) r^{2} \\
=\frac{\hbar^{2}}{2 m^{*}} \frac{1}{\Phi(\theta)} \frac{\partial^{2} \Phi(\theta)}{\partial \theta^{2}}
\end{gathered}
$$

the right part can be written as follows:

$$
\frac{\hbar^{2}}{2 m^{*}} \frac{1}{\Phi(\theta)} \frac{\partial^{2} \Phi(\theta)}{\partial \theta^{2}}=\frac{-\hbar^{2}}{2 m^{*}} n^{2}
$$

where $n$ is the orbital quantum number that must be integer:

$$
\frac{-\hbar^{2}}{2 m^{*}}\left(\frac{r^{2}}{\chi(\mathrm{r}, \mathrm{z})} \frac{\partial^{2} \chi(\mathrm{r}, \mathrm{z})}{\partial r^{2}}+\frac{r}{\chi(\mathrm{r}, \mathrm{z})} \frac{\partial \chi(\mathrm{r}, \mathrm{z})}{\partial r}++\frac{\partial^{2} \chi(\mathrm{r}, \mathrm{z})}{\partial z^{2}}\right)+(V-E) r^{2}+\frac{\hbar^{2}}{2 m^{*}} n^{2}=0
$$

when multiplying this equation by $\chi(r, z) / r^{2}$, we find the following equation:

$$
\begin{gathered}
-\frac{\hbar^{2}}{2 m^{*}}\left(\frac{\partial^{2} \chi(r, z)}{\partial r^{2}}+\frac{\partial^{2} \chi(r, z)}{\partial z^{2}}\right)+\left(V+\frac{1}{r^{2}} \frac{\hbar^{2}}{2 m^{*}} n^{2}\right) \chi(r, z)-\frac{\hbar^{2}}{2 m^{*}} \frac{1}{r} \frac{\partial \chi(r, z)}{\partial r} \\
=E \chi(r, z)
\end{gathered}
$$

To calculate the energy states and theirs wave functions for electron/hole in the QD, Dirichlet conditions are applied where the wave function must be zero (outside the considered domain) and the Neumann conditions are employed for the internal boundaries to ensure the continuity of the wave function. One of the major advantages of FEM is the flexibility to vary the desired precision over the 
entire domain by controlling the quality of the mesh. Accordingly, an adaptive mesh refinement has been employed as shown by the Figure 1 .

The impact of strain on the carrier confinement due to the lattice mismatch between InAs and GaAs materials is also taken into account for the calculation [20]. Hydrostatic and uniaxial strains are given by the following expressions:

$$
\epsilon_{h}=\epsilon_{x x}+\epsilon_{y y}+\epsilon_{z z}
$$

and

$$
\epsilon_{b}=\epsilon_{z z}-\frac{1}{2}\left(\epsilon_{x x}+\epsilon_{y y}\right)
$$

where $\varepsilon_{b}, \varepsilon_{h}$ are hydrostatics and uniaxial strain respectively. The lattice mismatch between InAs and GaAs is given by:

$$
\epsilon_{x x}=\epsilon_{y y}=\frac{a_{G a A s}-a_{I n A s}}{a_{I n A s}}
$$

and

$$
\epsilon_{z z}=-2 \frac{C_{12}}{C_{11}} \epsilon_{x x}
$$

The strained effect on conduction band is $\delta E_{c}=a_{c} \epsilon_{h}$ and for valence band is

$$
\delta E_{v}=a_{v} \epsilon_{h}-\frac{1}{2} b \epsilon_{b}
$$

then the strained band gap energy $(E g)$ can be written as:

$$
\mathrm{Eg}_{\text {InAs-str }}=\mathrm{Eg}_{\text {InAs-unstr }}+\delta E_{c}-\delta E_{v}
$$

where Eg InAs-unstr is the unstrained InAs band gap energy and $E g_{\text {InAs-str }}$ presents the strained one. The parameters used in this numerical approach are summarized in Table 1 [28].

Table 1. InAs and GaAs parameters used for the calculation.

\begin{tabular}{ccccccc}
\hline Material & $\mathbf{m}_{\mathbf{e}}{ }^{*}\left(\mathbf{m}_{\mathbf{0}}\right)$ & $\mathbf{m}_{\mathbf{h}}{ }^{*}\left(\mathbf{m}_{\mathbf{0}}\right)$ & $\begin{array}{c}\mathbf{C}_{\mathbf{1 1}} \mathbf{1 0}^{\mathbf{1 1}} \mathbf{( \mathbf { d y n }} \\
\left.\mathbf{c m}^{-\mathbf{2}}\right)\end{array}$ & $\mathbf{C}_{\mathbf{1 2}} \mathbf{1 0}^{\mathbf{1 1}} \mathbf{( \mathbf { d y n } \mathbf { c m } ^ { - \mathbf { 2 } } )}$ & $\left(\boldsymbol{a}_{\mathbf{c}}-\boldsymbol{a}_{\mathbf{v}}\right)(\mathbf{e V})$ & $\mathbf{b}(\mathbf{e V})$ \\
\hline $\mathrm{InAs}$ & 0.023 & 0.41 & 8.32 & 4.52 & -4.08 & -1.8 \\
$\mathrm{GaAs}$ & 0.068 & 0.5 & 12.21 & 5.66 & -6 & -2 \\
\hline
\end{tabular}

The single particle optical transition energies $\mathrm{E}_{i}$ can be deduced by the following equation:

$$
E_{i}=e_{i}+E g_{\text {InAs-Str }}+h_{i}
$$

where $e_{i}\left(h_{i}\right)$ correspond to the electron (hole) confined state.

Figure 2 shows the high excitation power $12 \mathrm{~K} \mathrm{PL}$ data recorded for the reference sample where the QDs are in a pure GaAs matrix. The appearance of multiple peaks results from the states filling effects. The ground state (GS) emission energy is located around $1.086 \mathrm{eV}$ with two excited states at $1.155 \mathrm{eV}$ and $1.224 \mathrm{eV}$. The appearance of well resolved optical transition energies allows us to estimate the mean size of the buried QDs. Indeed, by tuning the QDs AR, it is possible to fit the calculated ground and excited states transition energies to the experimentally measured PL emission energies. 


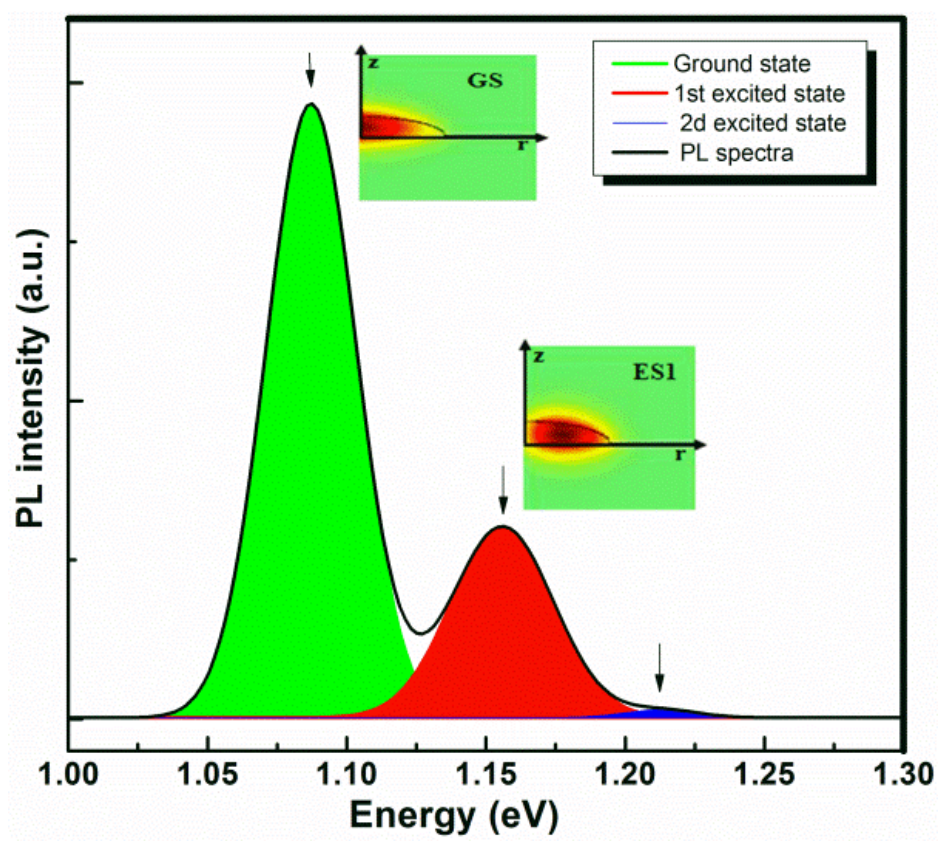

Figure 2. $12 \mathrm{~K}$ photoluminescence spectroscopy (PL) spectra taken with an excitation power of $400 \mathrm{~mW}$, from the reference InAs QDs in a pure GaAs matrix showing a ground state and two excited states emission peaks. The arrows indicate the calculated transition energies. The electron envelope functions are also shown as insets.

The calculation has been based on the three emission energies appearing in the PL spectrum. The experimental and calculated emission energies are perfectly matched within some meV yielding an average QD height of $3.8 \mathrm{~nm}$ and a base diameter around $28 \mathrm{~nm}$. The calculated wave functions of the first two confined electron states using the estimated realistic size for the buried QDs with an InAs wetting layer thickness of $0.3 \mathrm{~nm}$ are shown in the Figure 2. It is important to notice that atomic force microscopy (AFM) investigation of similar uncapped structure revealed a surface density around $2.4 \times 10^{10} \mathrm{~cm}^{-2}$ and a mean dots' height of $8 \mathrm{~nm}$ with a base diameter around $35 \mathrm{~nm}$ giving rise to an aspect ratio (AR) of 0.23 . This shows that the values are over estimated. Indeed, the QDs height can be reduced by the capping process [29,30]. Additionally, the AFM technique may also provide an over estimated size due to the tip artifact [31,32]. It is also reasonable to take into account the fact that assuming a pure InAs QDs in the calculation might yields smaller size QDs than the real case where small amount of In atoms may out diffuse.

In order to study the impact the QDs position with respect to the InGaAs SRL, we have conducted a first investigation using these reference QDs parameters. This implies that the InGaAs capping or underlying layer will contribute to the strain reduction without affecting the QDs size. The calculations expect a ground state emission energy redshift around $20 \mathrm{meV}$ upon capping the InAs QDs by $10 \mathrm{~nm}$ of InGaAs and around $10 \mathrm{meV}$ when the SRL is inserted below the QDs.

The PL spectra show that capping the QDs by $10 \mathrm{~nm}$ (QDCL) leads to a decrease of the PL emission energy by approximately $32 \mathrm{meV}$ as shown by the Figure 3. 


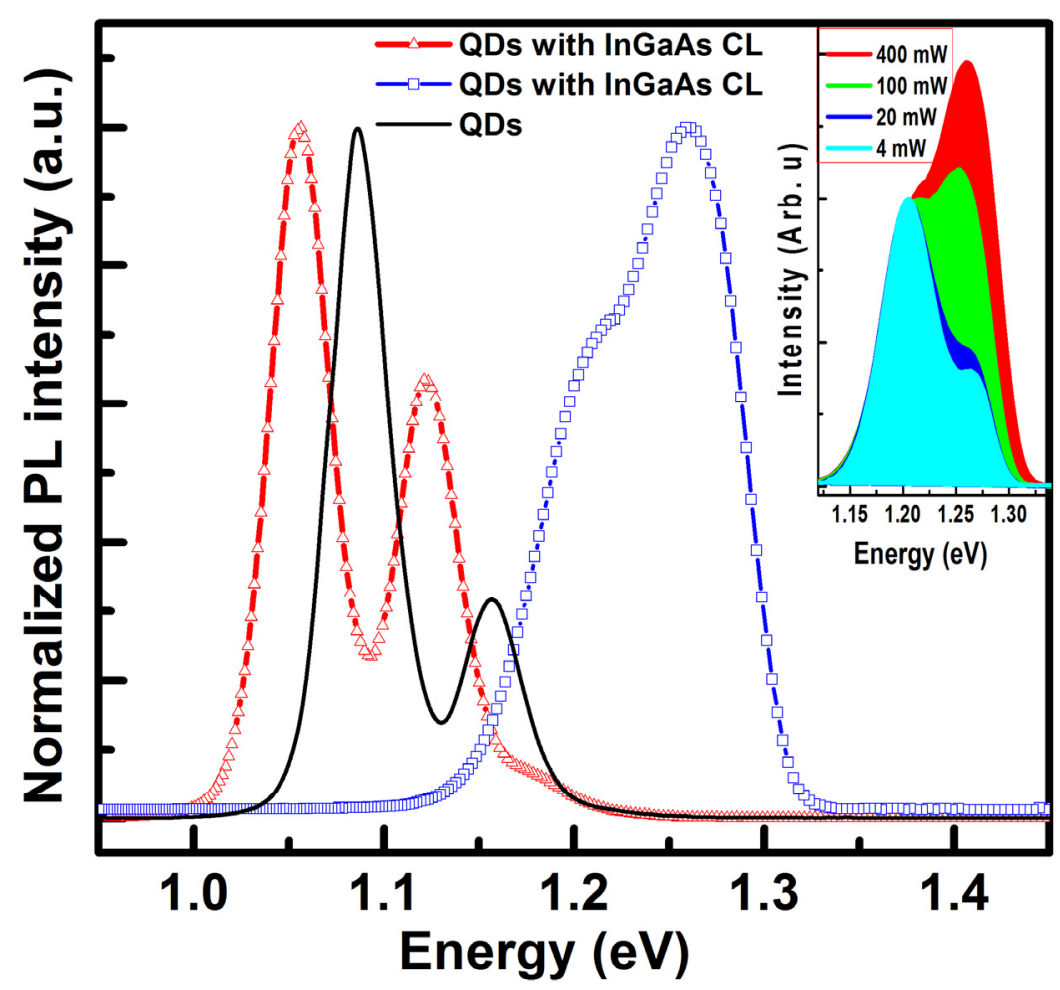

Figure 3. $12 \mathrm{~K}$ PL spectra taken with an excitation power of $400 \mathrm{~mW}$, from the reference InAs QDs in a pure GaAs matrix and from the InAs QDs with SRL as a cap layer (empty triangles) and as an underlying layer (empty squares). The Inset shows the excitation power dependent PL spectra from the InAs QDs with InGaAs underlying layer. Multiple peaks in each spectra arise from the stat filling effects.

The excess energy red shift could arise from the increase of the QDs' size a result of the strain driven phase separation of the capping layer alloy. This result is in agreement with recently reported structural investigation on similar structures [23]. In the meanwhile, the GS emission energy of the InAs QDs with InGaAs underlying layer (QDUL) shows an unexpected blueshift of the GS emission energy up to $1.204 \mathrm{eV}$ with broadening of the PL linewidth and reduction of the intersublevel spacing energy. A shown by the inset of the Figure 3, the persistence of the excited states emission energy peak at the lower excitation power, testify to a reduced number of coherent QDs [11]. Furthermore, AFM investigation on similar uncapped InAs QDs revealed an increased dots surface density to $4 \times 10^{10} \mathrm{~cm}^{-2}$, with a broadened size distribution a reduced mean dots' height down to $5 \mathrm{~nm}$ without appearance of bimodal size distribution [11] confirming the later statement. These observations indicate that the mean dots' size has been seriously altered by the InGaAs underlying layer.

In fact, by varying the dots' size we succeed in accurately fitting the PL emission energies by the calculated values for both InAs QDs with either SRL capping or underlying layer. The results are summarized in Table 2, where the estimated results using the reference QDs size are shown together with the simulated real dots' size obtained by tuning the dots' size to match the experimental transition energies. Accordingly, the calculation reveals an increased buried dots' size upon capping by InGaAs SRL, while keeping the same aspect ratio. This change of InAs QDs size is explained by the redistribution of Indium and Gallium atoms during the deposition of the InGaAs capping layer on InAs QDs resulted from alloy phase separation phenomena [16]. 
Table 2. Experimental and theoretical Ground state and first two excited states optical transition energies as well as corresponding QD size. The experimental emission energies are given by PL spectroscopy and the average QDs size is estimated through AFM. The expected results refer to the calculated transition energies keeping the simulated buried dot size for the reference sample. The Simulation refers to the realistic dots' size driven by fitting the theoretical emission energies to the corresponding PL peaks.

\begin{tabular}{ccccccc}
\hline Sample & Data & $\mathbf{H}_{\mathbf{Q D}} / \mathbf{D}_{\mathbf{Q D}}$ & Aspect Ratio $(\boldsymbol{\alpha})$ & $\mathbf{E}_{\mathbf{0}}(\mathbf{e V})$ & $\mathbf{E}_{\mathbf{1}}(\mathbf{e V})$ & $\mathbf{E}_{\mathbf{2}}(\mathbf{e V})$ \\
\hline \multirow{2}{*}{ QDs } & Experiments & $8.5 / 35$ & 0.24 & 1.086 & 1.156 & 1.224 \\
& Simulation & $3.8 / 28$ & 0.13 & 1.083 & 1.151 & 1.238 \\
& Experiments & $8.5 / 35$ & 0.24 & 1.054 & 1.117 & 1.194 \\
QDCL & Simulation & $4 / 30$ & 0.13 & 1.051 & 1.113 & 1.193 \\
& Expected results & $3.8 / 28$ & 0.13 & 1.068 & 1.131 & 1.213 \\
& Experiments & $5 / 41$ & 0.12 & 1.21 & 1.261 & - \\
QDUL & Simulation & $2.5 / 29$ & 0.08 & 1.204 & 1.265 & 1.331 \\
& Expected results & $3.8 / 28$ & 0.13 & 1.071 & 1.141 & 1.217 \\
\hline
\end{tabular}

However, a drastic change of the mean size and aspect ratio is found to occur for the QDs with InGaAs underlying layer. Indeed, the dots' height is found to decrease to $2.5 \mathrm{~nm}$ and the base diameter is increased to $29 \mathrm{~nm}$. In fact, indium segregation during the growth of the SRUL is expected to increase the nominal thickness of InAs material from 2.4 ML to up to $3 \mathrm{ML}$. Indeed, when the InAs nominal thickness exceeds a critical value, coalesced or/and dislocated QDs are formed and the number and size of active QDs could be critically reduced. This effect is confirmed by detailed PL investigation indicating a degradation of the QDs structural properties [11]. Additionally, the reduced strain increases the InAs critical thickness which is favorable to the formation of thicker wetting layers allowing the growth of smaller size QDs with higher areal density [33].

To further investigate the effect of the size modification that occurred following the growth of the QDs on the top of the InGaAs layer, the calculated electrons wave functions for the ground state and the first excited state are shown by the Figure 4 in the $(r, z)$ plan.
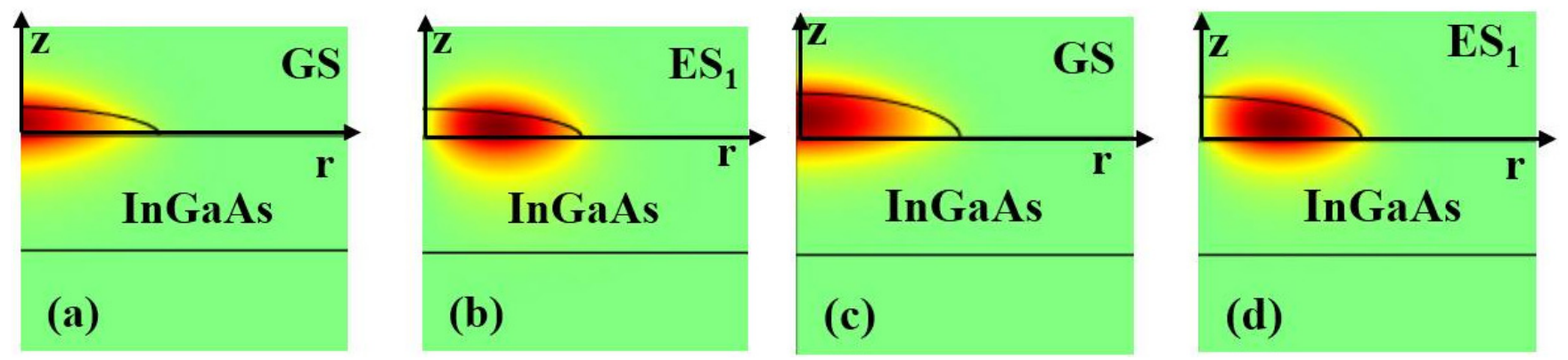

Figure 4. (a) and (b) indicates the envelop function for electrons with the simulated real size of the buried dots; (c) and (d) refer to the envelop functions expected for QDs with InGaAs underlying layer (QDUL) with a dot-size that is supposed to be the same as the reference sample.

Indeed, as a consequence of the decreased realistic size, compared to that of the reference QDs, the electron wave function is found to spread out of the QDs. This behavior indicates less localization 
as a consequence of the lowering of the effective confining potential barrier and increased confined energy levels.

\section{Experimental Section}

For experimental validation and deeper investigation of the effects of the InGaAs layer's position on the structural and optical properties of InAs QDs we have used a single InAs QDs layer as reference sample. In the two other samples, the strain has been reduced by an InGaAs layer with $15 \%$ indium composition. In the QDCL sample, the QDs were covered by $10 \mathrm{~nm}$ InGaAs. However, in the QDUL sample, the InAs QDs layer was deposited on a $10 \mathrm{~nm}$ InGaAs. The samples are characterized by PL spectroscopy using a conventional lock-in technique. A $514.5 \mathrm{~nm}$ line of an Ar + laser with $0.2 \mathrm{~mm}$ spot diameter has been employed as excitation source. More details on the growth process, optical and morphological properties of these samples can be found elsewhere [11].

\section{Conclusions}

In summary, a combination of a simple numerical approach in the frame of the effective mass approximation method and photoluminescence spectroscopy have been employed to perform a detailed investigation of the effect of the InAs QDs position with respect to the strain reducing layer on the buried dots' size. The numerical calculations using the same dot-size expects a redshift of the emission energies upon underlaying or capping the dots by InGaAs alloy. The PL results show additional redshift for the emission energy of the InGaAs capped QDs and a realistic QDs size simulation revealed an enhancement induced by the alloy phase separation. When the QDs are grown on the top of the InGaAs SRL, the experiments show an unexpected blueshift. The simulation of the buried dots' size indicated a drastic reduction of the QDs' height leading to less carrier localization. Our results provide insight towards better understanding and optimization of QDs' properties by using SRL.

\section{Acknowledgments}

The last author (Bouroui Ilahi) would like to extend his sincere appreciation to the Deanship of Scientific Research at king Saud University for funding this Research group NO: RG-1436-014.

\section{Author Contributions}

Manel Souaf and Mourad Baira did the calculations, Olfa Nasr and Mohamed Helmi Hadj Alouane has performed the Photoluminescence measurements, Larbi Sfaxi and Hassen Maaref did the samples growth. Bouraoui Ilahi has planned the work and contributed to the characterization and numerical calculation. All the co-authors have contributed to the revision of the paper written by Manel Souaf.

\section{Conflicts of Interest}

The authors declare no conflict of interest. 


\section{References}

1. Majid, M.A.; Childs, D.T.D.; Kennedy, K.; Airey, R.; Hogg, R.A.; Clarke, E.; Spencer, P.; Murray, R. O-band excited state quantum dot bilayer lasers. App. Phys. Lett. 2011, 99, 051101.

2. Ustinov, V.M.; Zhukov, A.E. GaAs-based long-wavelength lasers. Semicond. Sci. Technol. 2000, $15,41-54$.

3. Salter, C.L.; Stevenson, R.M.; Farrer, I.; Nicoll, C.A.; Ritchie, D.A.; Shields, A.J. Single-photon and entangled-photon sources for quantum information. Nature 2010, 465, 594-597.

4. Shields, A.J.; O’Sullivan, M.P.; Farrer, I.; Ritchie, D.A.; Hogg, R.A.; Leadbeater, M.L.; Norman, C.E.; Pepper, M. Detection of single photons using a field-effect transistor gated by a layer of quantum dots. Appl. Phys. Lett. 2000, 76, 3673-3675.

5. Ledentsov, N.N. Quantum dot laser. Semicond. Sci. Technol. 2011, 26, 014001.

6. Chen, J.X.; Markus, A; Fiore, A.; Oesterle, U.; Stanley, R.P.; Carlin, J.F.; Houdré, R.; Ilegems, M.; Lazzarini, L.; Nasi, L.; et al. Tuning InAs/GaAs quantum dot properties under Stranski-Krastanov growth mode for $1.3 \mu \mathrm{m}$ applications. J. Appl. Phys. 2002, 91, 6710-6716.

7. Sogabe, T.; Shoji, Y.; Ohba, M.; Yoshida, K.; Tamaki, R.; Hong, H.F.; Wu, C.H.; Kuo, C.T.; Tomić, S.; Okada, Y. Intermediate-band dynamics of quantum dots solar cell in concentrator photovoltaic modules. Sci. Rep. 2014, 4, doi:10.1038/srep04792.

8. Kong, L.; Sun, W.; Feng, Z.C.; Xie, S.; Zhou, Y.; Wang, R.; Zhang, C.; Zong, Z.; Wang, H.; Qiao, Q.; et al. Effects of InAlAs strain reducing layer on the photoluminescence properties of InAs quantum dots embedded in InGaAs/GaAs quantum wells. Thin Solid Films 2014, 562, 440-444.

9. Liu, H.Y.; Hopkinson, M.; Harrison, C.N.; Steer, M.J.; Frith, R.; Sellers, I.R.; Mowbray, D.J.; Skolnick, M.S. Optimizing the growth of $1.3 \mu \mathrm{m}$ InAs/InGaAs dots-in-a-well structure. J. Appl. Phys. 2003, 93, 2931-2936.

10. Seravalli, L.; Minelli, M.; Frigeri, P.; Franchi, S.; Guizzetti, G.; Patrini, M.; Ciabattoni, T.; Geddo, M. Quantum dot strain engineering of InAs/InGaAs nanostructures. J. Appl. Phys. 2007, 101, doi:10.1063/1.2424523.

11. Nasr, O.; HadjAlouane, M.H.; Maaref, H.; Hassen, F.; Sfaxi, L.; Ilahi, B. Comprehensive investigation of optical and electronic properties of tunable InAs QDs optically active at O-band telecommunication window with (In)GaAs surrounding material. J. Lumin. 2014, 148, 243-248.

12. Ustinov, V.M.; Maleev, N.A.; Zhukov, A.E.; Kovsh, A.R.; Egorov, A.Yu.; Lunev, A.V.; Volovik, B.V.; Krestnikov, I.L.; Musikhin, Yu.G.; Bert, N.A.; et al. InAs/InGaAs quantum dot structures on GaAs substrates emitting at $1.3 \mu \mathrm{m}$. Appl. Phys. Lett. 1999, 74, 1384-1385.

13. Nishi, K.; Saito, H.; Sugou, S.; Lee, J.S. A narrow photoluminescence linewidth of $21 \mathrm{meV}$ at 1.35 $\mu \mathrm{m}$ from strain-reduced InAs quantum dots covered by $\mathrm{In}_{0.2} \mathrm{Ga}_{0.8}$ as grown on GaAs substrates. Appl. Phys. Lett. 1999, 74, 1111-1113.

14. Pires, M.P.; Landi, S.M.; Tribuzy, C.V.B.; Nunes, L.A.; Marega, E.; Souza, P.L. InAs quantum dots over InGaAs for infrared photodetectors. J. Cryst. Growth 2004, 272, 192-197.

15. Chen, R.; Liu, H.Y.; Sun, H.D. Electronic energy levels and carrier dynamics in InAs/InGaAs dots-in-a-well structure investigated by optical spectroscopy. J. Appl. Phys. 2010, 107, doi:10.1063/1.3277049. 
16. Joyce, P.B.; Kryzewski, T.J.; Bell, G.R.; Joens, T.S.; le Ru, E.C.; Murray, R. Optimizing the growth of $1.3 \mu \mathrm{m}$ InAs/GaAs quantum dots. Phys. Rev. B 2001, 64, 235317.

17. Le Ru, E.C.; Howe, P.; Joens, T.S.; Murray, R. Strain-engineered InAs/GaAs quantum dots for long-wavelength emission. Phys. Rev. B 2003, 67, 165303.

18. Nabetani, Y.; Matsumoto, T.; Sasikala, G.; Suemune, I. Theory of strain states in InAs quantum dots and dependence on their capping layers. J. Appl. Phys. 2005, 98, 063502.

19. Ilahi, B.; Sfaxi, L.; Hassen, F.; Maaref, H.; Salem, B.; Guillot, G.; Jbeli, A.; Marie, X. Optical properties of $1.3 \mu \mathrm{m}$ room temperature emitting InAs quantum dots covered by $\mathrm{In}_{0.4 \mathrm{Ga} 0.6 \mathrm{As} / \mathrm{GaAs}}$ hetero-capping layer. Appl. Phys. A 2005, 81, 813-816.

20. Cherkashin, N.; Reboh, S.; Htch, M.J.; Claverie, A.; Preobrazhenskii, V.V.; Putyato, M.A.; Semyagin, B.R.; Chaldyshev, V.V. Determination of stress, strain, and elemental distribution within In(Ga)As quantum dots embedded in GaAs using advanced transmission electron microscopy. Appl. Phys. Lett. 2013, 102, 173115.

21. Ilahi, B.; Sfaxi, L.; Maaref, H. Optical investigation of InGaAs-capped InAs quantum dots: Impact of the strain-driven phase separation and dependence upon post-growth thermal treatment. J. Lumin. 2007, 127, 741-746.

22. Maia, A.D.B.; da Silva, E.C.F.; Quivy, A.A.; Bindilatti, V.; de Aquino, V.M.; Dias, I.F.L. Simulation of the electronic properties of $\operatorname{In}_{x} \mathrm{Ga}_{1-x}$ As quantum dots and their wetting layer under the influence of indium segregation. J. Appl. Phys. 2013, 114, 083708.

23. Wang, P.; Bleloch, A.L.; Falke, M.; Goodhew, P.J.; Ng, J.; Missous, M. Direct measurement of composition of buried quantum dots using aberration-corrected scanning transmission electron microscopy. Appl. Phys. Lett. 2006, 89, 072111.

24. García, J.M.; Medeiros-Ribeiro, G.; Schmidt, K.; Ngo, T.; Feng, J.L.; Lorke, A.; Kotthaus, J.; Petroff, P.M. Intermixing and shape changes during the formation of InAs self-assembled quantum dots. Appl. Phys. Lett. 1997, 74, 2014-2016.

25. Joyce, P.B.; Krzyzewski, T.J.; Bell, G.R.; Jones, T.S. Surface morphology evolution during the overgrowth of large InAs-GaAs quantum dots. Appl. Phys. Lett. 2001, 79, 3615-3617.

26. Kegel, I.; Metzger, T.H.; Lorke, A.; Peisl, J.; Stangl, J.; Bauer, G.; Nordlund, K.; Schoenfeld, W.V.; Petroff, P.M. Determination of strain fields and composition of self-organized quantum dots using x-ray diffraction. Phys. Rev. B 2001, 63, 035318.

27. COMSOL Multiphysics. Available online: http://www.comsol.com (accessed on 17 July 2015).

28. Vurgaftman, I.; Meyer, J.R.; Ram-Mohan, L.R. Band parameters for III-V compound semiconductors and their alloys. J. Appl. Phys. 2001, 89, 5815-5875.

29. Grossi, D.F.; Smereka, P.; Keizer, J.G.; Ulloa, J.M.; Koenraad, P.M. Height control of self-assembled quantum dots by strain engineering during capping. Appl Phys. Lett. 2014, 105, 143104.

30. Kim, J.S.; Yu, P.W.; Lee, J.S.; Kim, J.S.; Kim, S.G.; Leem, J.; Jeon, M. Height-controlled InAs quantum dots by using a thin InGaAs layer. Appl. Phys. Lett. 2002, 80, 4714-4716.

31. Shiramine, K.; Muto, S.; Shibayama, T.; Sakaguchi, N.; Ichinose, H.; Kozaki, T.; Sato, S.; Nakata, Y.; Yokoyama, N.; Taniwaki, M. Tip artifact in atomic force microscopy observations of InAs quantum dots grown in Stranski-Krastanow mode. J. Appl. Phys. 2007, 101, 033527. 
32. Gong, Q.; Liang, J.B.; Xu, B.; Ding, D.; Li, H.X.; Jiang, C.; Zhou, W.; Liu, F.Q.; Wang, Z.G.; Qiu, X.H.; et al. Analysis of atomic force microscopic results of InAs islands formed by molecular beam epitaxy. J. Cryst. Growth 1998, 192, 376-380.

33. Simmonds, P.J.; Sun, M.; Laghumavarapu, R.B.; Liang, B.; Norman, A.G.; Luo, J.W.; Huffaker, D.L. Improved quantum dot stacking for intermediate band solar cells using strain compensation. Nanotechnology 2014, 25, 445402.

(C) 2015 by the authors; licensee MDPI, Basel, Switzerland. This article is an open access article distributed under the terms and conditions of the Creative Commons Attribution license (http://creativecommons.org/licenses/by/4.0/). 